Journal of Mathematics and Informatics

Vol. 10, 2017, 83-88

ISSN: 2349-0632 (P), 2349-0640 (online)

Published 11 December 2017

www.researchmathsci.org

DOI: http://dx.doi.org/10.22457/jmi.v10a11

Journal of

Mathematics and

Informatics

\title{
Evaluation of Some Centered Polygonal Numbers by Using the Division Algorithm
}

\author{
V. Pandichelvi ${ }^{1}$ and P. Sivakamasundari ${ }^{2}$ \\ ${ }^{1}$ Department of Mathematics, Urumu Dhanalakshmi College \\ Trichy, Tamilnadu, India \\ e-mail: mvpmahesh2017@gmail.com \\ ${ }^{2}$ Department of Mathematics, BDUCC, Lalgudi, Trichy, India. \\ e-mail: kaneeska.s@gmail.com
}

Received 2 November 2017; accepted 7 December 2017

Abstract. In this communication, we find centered square number, centered octagonal number, centered dodecagonal number, centered hexadecagonal number and tetradecagonal number by using division algorithm.

Keywords: Division algorithm, centered polygonal numbers

AMS Mathematics Subject Classification (2010): 11D99

\section{Introduction}

In [1] a function $A: N \rightarrow N$ is defined by $A(n)=m$ where $m$ is the smallest natural number such that $n$ divides $m+\sum_{k=1}^{n} k^{2}$. In [2] a function $A(n)$ such that $A(n)=m$ where $m$ is the smallest natural number such that $n$ divides $m+\sum_{k=1}^{n} k$ and $m+\sum_{k=1}^{n} k^{3}$ are given. In this communication, we obtain a function $A(n)$ such that $A(n)=m$ where $m$ is some centered polygonal numbers, such that $2 P P_{n}$ divides $m+($ a cubical polynomial $)$

\section{Notations:}

$P P_{n}=\frac{1}{2}\left(n^{3}+n\right)$ be a pentagonal pyramidal number of rank $n$

$C S_{n}=2 n^{2}-2 n+1$ be a centered square number of rank $n$

$C O_{n}=4 n^{2}-4 n+1$ be a centered octagonal number of rank $n$ 
V. Pandichelvi and P. Sivakamasundari

$C D_{n}=6 n^{2}-6 n+1$ be a centered dodecagonal number of rank $n$

$C T_{n}=7 n^{2}-7 n+1$ be a centered tetradecagonal number of rank $n$

$\mathrm{CH}_{n}=8 n^{2}-8 n+1$ be a centered Hexadecagonal number of rank $n$

\section{Method of analysis}

\section{SECTION A: Evaluation of centered square number}

Let $A: N \rightarrow N$ be defined by $A(n)=m$ where $m$ is the smallest natural number such that $2 P P_{n}$ divides $m+\left(n^{3}-2 n^{2}+3 n-1\right)$. If $2 P P_{n}$ divides $\left(n^{3}-2 n^{2}+3 n-1\right)$, then $A(n)=2 n^{2}-2 n+1$, otherwise $A(n)=\left(2 n^{2}-2 n+1\right)-r$ where $r$ is the least non negative remainder when $\left(n^{3}-2 n^{2}+3 n-1\right)$ is divided by $2 P P_{n}$. Hence $A$ is defined for all $n$. By division algorithm such remainder is given by $\left(n^{3}-2 n^{2}+3 n-1\right)-2 q P P_{n}$ where $q$ is the quotient when $\left(n^{3}-2 n^{2}+3 n-1\right)$ is divided by $2 P P_{n}$ and is given by the greatest integer function of $\frac{\left(n^{3}-2 n^{2}+3 n-1\right)}{2 P P_{n}}$,

That is $q=\left[\frac{n^{3}-2 n^{2}+3 n-1}{2 P P_{n}}\right]$

Hence,

$$
\begin{aligned}
& A(n)=n+n^{3}-\left\{\left(n^{3}-2 n^{2}+3 n-1\right)-\left[\frac{\left(n^{3}-2 n^{2}+3 n-1\right)}{n+n^{3}}\right] \times n+n^{3}\right\} \\
& A(n)=n+n^{3}-n^{3}+2 n^{2}-3 n+1+\left[\left(\frac{n+n^{3}}{n+n^{3}}\right)-\frac{\left(2 n^{2}-2 n+1\right)}{n+n^{3}}\right] \times n+n^{3} \\
& A(n)=2 n^{2}-2 n+1+\left[1-\frac{\left(2 n^{2}-2 n+1\right)}{n+n^{3}}\right] \times n+n^{3} \\
& A(n)=2 n^{2}-2 n+1+0\left(\because 0<\frac{2 n^{2}-2 n+1}{n+n^{3}}<1\right)
\end{aligned}
$$

$A(n)=2 n^{2}-2 n+1=$ centered square number.

\section{SECTION B: Evaluation of centered octagonal number}

Let $A: N \rightarrow N$ be defined by $A(n)=m$ where $m$ is the smallest natural number such that $2 P P_{n}$ divides $m+\left(2 n^{3}-4 n^{2}+6 n-1\right)$. If $2 P P_{n}$ divides $\left(2 n^{3}-4 n^{2}+6 n-1\right)$, then $A(n)=4 n^{2}-4 n+1$, otherwise $A(n)=\left(4 n^{2}-4 n+1\right)-r$ where $r$ is the least non 
Evaluation of Some Centered Polygonal Numbers by Using the Division Algorithm negative remainder when $\left(2 n^{3}-4 n^{2}+6 n-1\right)$ is divided by $2 P P_{n}$. Hence $A$ is defined for all $n$. By division algorithm such remainder is given by $\left(2 n^{3}-4 n^{2}+6 n-1\right)-2 q P P_{n}$ where $q$ is the quotient when $\left(2 n^{3}-4 n^{2}+6 n-1\right)$ is divided by $2 P P_{n}$ and is given by the greatest integer function of $\frac{\left(2 n^{3}-4 n^{2}+6 n-1\right)}{2 P P_{n}}$, That is $q=\left[\frac{2 n^{3}-4 n^{2}+6 n-1}{2 P P_{n}}\right]$.

Hence,

$$
\begin{aligned}
& A(n)=n+n^{3}-\left\{\left(2 n^{3}-4 n^{2}+6 n-1\right)-\left[\frac{\left(2 n^{3}-4 n^{2}+6 n-1\right)}{n+n^{3}}\right] \times n+n^{3}\right\} \\
& A(n)=n+n^{3}-2 n^{3}+4 n^{2}-6 n+1+\left[\left(\frac{n+n^{3}}{n+n^{3}}\right)+\frac{n^{3}-4 n^{2}+5 n-1}{n+n^{3}}\right] \times n+n^{3} \\
& A(n)=-n^{3}+4 n^{2}-5 n+1+\left[1+\frac{n^{3}-4 n^{2}+5 n-1}{n+n^{3}}\right] \times n+n^{3} \\
& A(n)=-n^{3}+4 n^{2}-5 n+1+n+n^{3}\left(\because 0<\frac{n^{3}-4 n^{2}+5 n-1}{n+n^{3}}<1\right)
\end{aligned}
$$

$A(n)=4 n^{2}-4 n+1=$ centered octagonal number.

\section{SECTION C : Evaluation of centered dodecagonal number}

Let $A: N \rightarrow N$ be defined by $A(n)=m$ where $m$ is the least natural number such that $2 P P_{n}$ divides $m+\left(3 n^{3}-6 n^{2}+9 n-1\right)$. If $2 P P_{n}$ divides $\left(3 n^{3}-6 n^{2}+9 n-1\right)$, then $A(n)=6 n^{2}-6 n+1$, otherwise $A(n)=\left(6 n^{2}-6 n+1\right)-r$ where $r$ is the least non negative remainder when $\left(3 n^{3}-6 n^{2}+9 n-1\right)$ is divided by $2 P P_{n}$. Hence $A$ is defined for all $n$. By division algorithm such residue is given by $\left(3 n^{3}-6 n^{2}+9 n-1\right)-2 q P P_{n}$ where $q$ is the quotient when $\left(3 n^{3}-6 n^{2}+9 n-1\right)$ is divided by $2 P P_{n}$ and is given by the greatest integer function of $\frac{\left(3 n^{3}-6 n^{2}+9 n-1\right)}{2 P P_{n}}$,

That is $q=\left[\frac{3 n^{3}-6 n^{2}+9 n-1}{2 P P_{n}}\right]$.

Hence, 
V. Pandichelvi and P. Sivakamasundari

$$
\begin{aligned}
& A(n)=n+n^{3}-\left\{\left(3 n^{3}-6 n^{2}+9 n-1\right)-\left[\frac{\left(3 n^{3}-6 n^{2}+9 n-1\right)}{n+n^{3}}\right] \times n+n^{3}\right\} \\
& A(n)=n+n^{3}-3 n^{3}+6 n^{2}-9 n+1+\left[\left(\frac{2\left(n+n^{3}\right)}{n+n^{3}}\right)+\frac{n^{3}-6 n^{2}+7 n-1}{n+n^{3}}\right] \times n+n^{3} \\
& \left.A(n)=-2 n^{3}+6 n^{2}-8 n+1+\left[2+\frac{n^{3}-6 n^{2}+7 n-1}{n+n^{3}}\right] \times n+n^{3}\right] \\
& A(n)=-2 n^{3}+6 n^{2}-8 n+1+2 n+2 n^{3}\left(\because 0<\frac{n^{3}-6 n^{2}+7 n-1}{n+n^{3}}<1\right) \\
& A(n)=6 n^{2}-6 n+1=\text { centered dodecagonal number. }
\end{aligned}
$$

\section{Section D: Evaluation of tetradecagonal number}

Let $A: N \rightarrow N$ be defined by $A(n)=m$ where $m$ is the smallest natural number such that $2 P P_{n}$ divides $m+\left(2 n^{3}-7 n^{2}+9 n-1\right)$. If $2 P P_{n}$ divides $\left(2 n^{3}-7 n^{2}+9 n-1\right)$, then $A(n)=7 n^{2}-7 n+1$, otherwise $A(n)=\left(7 n^{2}-7 n+1\right)-r$ where $r$ is the least non negative remainder when $\left(2 n^{3}-7 n^{2}+9 n-1\right)$ is divided by $2 P P_{n}$. Hence $A$ is defined for all $n$. By division algorithm such remainder is given by $\left(2 n^{3}-7 n^{2}+9 n-1\right)-2 q P P_{n}$ where $q$ is the quotient when $\left(2 n^{3}-7 n^{2}+9 n-1\right)$ is divided by $2 P P_{n}$ and is given by the greatest integer function of $\frac{\left(2 n^{3}-7 n^{2}+9 n-1\right)}{2 P P_{n}}$,

That is $q=\left[\frac{2 n^{3}-7 n^{2}+9 n-1}{2 P P_{n}}\right]$.

Hence ,

$$
\begin{aligned}
& A(n)=n+n^{3}-\left\{\left(2 n^{3}-7 n^{2}+9 n-1\right)-\left[\frac{\left(2 n^{3}-7 n^{2}+9 n-1\right)}{n+n^{3}}\right] \times n+n^{3}\right\} \\
& A(n)=n+n^{3}-2 n^{3}+7 n^{2}-9 n+1+\left[\left(\frac{n+n^{3}}{n+n^{3}}\right)+\frac{\left(n^{3}-7 n^{2}+8 n-1\right)}{n+n^{3}}\right] \times n+n^{3} \\
& A(n)=-n^{3}+7 n^{2}-8 n+1+\left[1+\frac{\left(n^{3}-7 n^{2}+8 n-1\right)}{n+n^{3}}\right] \times n+n^{3}
\end{aligned}
$$


Evaluation of Some Centered Polygonal Numbers by Using the Division Algorithm $A(n)=-n^{3}+7 n^{2}-8 n+1+n+n^{3}\left(\because 0<\frac{\left(n^{3}-7 n^{2}+8 n-1\right)}{n+n^{3}}<1\right)$ $A(n)=7 n^{2}-7 n+1=$ centered tetradecagonal number.

\section{SECTION E: Evaluation of centered hexadecagonal number}

Let $A: N \rightarrow N$ be defined by $A(n)=m$ where $m$ is the lowest natural number such that $2 P P_{n}$ divides $m+\left(3 n^{3}-8 n^{2}+11 n-1\right)$. If $2 P P_{n}$ divides $\left(3 n^{3}-8 n^{2}+11 n-1\right)$, then $A(n)=8 n^{2}-8 n+1$, otherwise $A(n)=\left(8 n^{2}-8 n+1\right)-r$ where $r$ is the least non negative remainder when $\left(3 n^{3}-8 n^{2}+11 n-1\right)$ is divided by $2 P P_{n}$. Hence $A$ is defined for all $n$. By division algorithm such remainder is given by $\left(3 n^{3}-8 n^{2}+11 n-1\right)-2 q P P_{n}$ where $q$ is the quotient when $\left(3 n^{3}-8 n^{2}+11 n-1\right)$ is divided by $2 P P_{n}$ and is given by the greatest integer function of $\frac{\left(3 n^{3}-8 n^{2}+11 n-1\right)}{2 P P_{n}}$,

That is $q=\left[\frac{3 n^{3}-8 n^{2}+11 n-1}{2 P P_{n}}\right]$.

Hence,

$$
\begin{aligned}
& A(n)=n+n^{3}-\left\{\left(3 n^{3}-8 n^{2}+11 n-1\right)-\left[\frac{\left(3 n^{3}-8 n^{2}+11 n-1\right)}{n+n^{3}}\right] \times n+n^{3}\right\} \\
& A(n)=n+n^{3}-3 n^{3}+8 n^{2}-11 n+1+\left[\left(\frac{2\left(n+n^{3}\right)}{n+n^{3}}\right)+\frac{n^{3}-8 n^{2}+9 n-1}{n+n^{3}}\right] \times n+n^{3} \\
& A(n)=-2 n^{3}+8 n^{2}-10 n+1+\left[2+\frac{n^{3}-8 n^{2}+9 n-1}{n+n^{3}}\right] \times n+n^{3} \\
& A(n)=-2 n^{3}+8 n^{2}-10 n+1+2 n+2 n^{3}\left(\because 0<\frac{n^{3}-8 n^{2}+9 n-1}{n+n^{3}}<1\right)
\end{aligned}
$$

$A(n)=8 n^{2}-8 n+1=$ centered hexadecagonal number.

\section{Conclusion}

In this communication, we find the centered polygonal numbers through the divisibility algorithm. In this manner, one can find the some special numbers through the divisibility algorithm.

\section{REFERENCES}

1. A.W.Vyawahare, A new function on divisibility, The Mathematics Education, 
V. Pandichelvi and P. Sivakamasundari

XL (2006) 122-128.

2. M.A.Gopalan and V.Pandichelvi, Two remarkable functions on divisibility, Acta Ciencia Indica, XXXIV M (3) (2008) 1161.

3. van Nivan, Herbert, S.Zuckermann and L.Montgomery, An Introduction to Theory of Numbers, John Wiley and Sons Inc, New York 2004.

4. A.Choudhary, The general solution of a cubic Diophantine equation, Ganita, 50(1) (1999) 1-4.

5. A.H.Beiler, Ch.18 in Recreations in the Theory of Numbers, The Queen of Mathematics Entertains, New York, Dover, 1996. 